\title{
PRIME IDEALS OF QUANTIZED WEYL ALGEBRAS
}

\author{
by M. AKHAVIZADEGAN $\dagger$ and D. A. JORDAN
}

(Received 1 March, 1995)

1.1. Introduction. The main object of study in this paper is the quantized Weyl algebra $A_{n}^{\bar{q}, \Lambda}$ which arises from the work of Maltsiniotis [10] on noncommutative differential calculus. This algebra has been studied from the point of view of noncommutative ring theory by various authors including Alev and Dumas [1], the second author [9], Cauchon [3], and Goodearl and Lenagan [5]. In [9], it is shown that $A_{n}^{\bar{q}, \Lambda}$ has $n$ normal elements $z_{i}$ and, subject to a condition on the parameters, the localization $B_{n}^{\bar{q}, \Lambda}$ obtained on inverting these elements is simple of Krull and global dimension $n$. It is easy to show that each of these normal elements generates a height one prime ideal and that these are all the height one prime ideals of $A_{n}^{\bar{q}, \Lambda}$. The purpose of this paper is to determine, under a stronger condition on the parameters, all the prime ideals of $A_{n}^{\bar{q}, \Lambda}$ and to compare the prime spectrum with that of a related algebra $\mathscr{A}_{n}^{\bar{q}, \Lambda}$. This algebra has more symmetric defining relations than those of $A_{n}^{\bar{q}, \Lambda}$ but it shares the same simple localization $B_{n}^{\bar{q}, \Lambda}$, which again is obtained by inverting $n$ normal elements $z_{i}$. Like $A_{n}^{\bar{q}, \Lambda}$, the alternative algebra can be regarded as an algebra of skew differential (or difference) operators on the coordinate ring of quantum $n$-space.

The construction of both $A_{n}^{\bar{q}, \Lambda}$ and $\mathscr{A}_{n}^{\bar{q}, \Lambda}$ involves parameters $q_{i}, 1 \leq i \leq n$ and $\lambda_{j i}$, $1 \leq i<j \leq n$. Let $G(\Lambda, \bar{q})$ be the subgroup of the multiplicative group of the base field $k$ generated by these parameters. The conditions that we impose on the parameters are that the ranks of certain subgroups of $G(\Lambda, \bar{q})$ should be maximal. Under these conditions, in both $A_{n}^{\bar{q}, \Lambda}$ and $\mathscr{A}_{n}^{\bar{q}, \Lambda}$, every nonzero prime is generated by a normalizing sequence of generators and we give an explicit description of these sequences. However there are some significant differences between the two cases. In $\mathscr{A}_{n}^{\bar{q}, \Lambda}$, provided $n>1$, the spectrum is finite and every nonzero prime ideal is generated by a subsequence of the sequence $z_{1}, z_{2}, \ldots, z_{n}$. Thus the maximal length of a chain of prime ideals is $n$. On the other hand, in $A_{n}^{\bar{q}} \wedge$ there are always infinitely many maximal ideals and they have height $2 n$. However the number of nonmaximal prime ideals is finite. One interesting feature is that there is a unique prime ideal of height $2 n-1$. This is consistent with the known spectrum for the first quantized Weyl algebra $A q[4,8.4]$ and it is also closer in nature to that for the coordinate ring of quantum space under the analogous conditions on the parameters.

In the remainder of Section 1, we establish the basic terminology for the rings to be considered later in the paper. Sections 2, 3 and 4 will discuss the prime ideals of the coordinate ring of quantum space, of $\mathscr{A}_{n}^{\bar{q}, \Lambda}$ and $A_{n}^{\bar{q}, \Lambda}$ respectively. The results on $A_{n}^{\bar{q}, \Lambda}$ have been obtained independently by Rigal [14] under stronger conditions on the parameters. Although there are inevitable common features, Rigal's method, which is based on [7, Theorem 11.1], is essentially different. There are also features in common with the work of Goodearl and Lenagan [5] on catenarity and with the work of Oh [13] on the primitive ideals of the coordinate ring of quantum symplectic space.

† With the support of the Ministry of Culture and Higher Education of the Islamic Republic of Iran.

Glasgow Math. J. 38 (1996) 283-297. 
We thank the referee for some helpful suggestions, in particular, the inclusion of Corollary 3.5 .

1.2. Quantum $n$-space. Throughout, $k$ will be an algebraically closed field and $k^{*}$ will denote the multiplicative group of $k$.

Let $R$ be a $k$-algebra and let $r, s \in R$ and $q \in k^{*}$. We say that $r q$-commutes with $s$ if $r s=q s r$. More symmetrically, we say that $r$ and $s$ semicommute (with each other) if $r$ $q$-commutes with $s$ for some $q \in k^{*}$.

Let $\Lambda=\left[\lambda_{i j}\right]$ be an $n \times n$ matrix of nonzero elements of $k$ such that each $\lambda_{i i}=1$ and $\lambda_{i j}$ is always equal to $\lambda_{j i}^{-1}$. We denote by $A(\Lambda)$ the $k$-algebra generated by $n$ indeterminates $x_{i}, 1 \leq i \leq n$, subject to the semicommutation relations

$$
x_{j} x_{i}=\lambda_{j i} x_{i} x_{j}
$$

This algebra is now usually called the coordinate ring of quantum $n$-space. Each $x_{i}$ is normal in $A(\Lambda)$ and we denote by $P(\Lambda)$ the algebra obtained by inverting each $x_{i}$. These are the algebras considered in [11].

1.3. Iterated skew polynomial rings. Both $A_{n}^{\bar{q}, \Lambda}$ and $\mathscr{A}_{n}^{\bar{q}, \Lambda}$ arise from the construction of iterated skew polynomial rings studied in [9]. Here we describe this construction in the generality appropriate to this paper. For full details and for justification of the statements made below, see [9].

Let $A$ be a $k$-algebra with two commuting $k$-automorphisms, $\alpha$ and $\gamma$, such that there exists a nonzero normal element $v$ of $A$ with $v a=\gamma(a) v$ for all $a \in A$. Let $\beta=\alpha^{-1} \gamma$, let $S$ denote the skew polynomial ring $A[y ; \alpha]$ and extend $\beta$ to $S$ by setting $\beta(y)=\rho y$ for some nonzero $\rho \in k$. There is a $\beta$-derivation $\delta$ of $S$ such that $\delta(A)=0$ and $\delta(y)=v$. Let $R=R(A, \alpha, v, \rho)$ be the iterated skew polynomial ring $A[y ; \alpha][x ; \beta, \delta]$. Thus, for all $a \in A$,

$$
y a=\alpha(a) y, \quad x a=\beta(a) x, \quad x y-\rho y x=v .
$$

In this paper, we assume that $\rho \neq 1$ and also that $\alpha(v)=v$. The relation $x y-\rho y x=v$ can then be rewritten $x y-u=\rho(y x-u)$, where $u=\frac{1}{1-\rho} v$. The element

$$
z=x y-y x=v+(\rho-1) y x=\rho^{-1}(v+(\rho-1) x y)
$$

will be called the Casimir element of $R$. (This is a nonzero scalar multiple of the Casimir element $x y-u$ used in [9].) The following identities hold:

$$
z y=\rho y z, \quad z x=\rho^{-1} x z, \quad z a=\gamma(a) z \quad \forall a \in A .
$$

Consequently $z$ is a normal element of $R$ inducing a $k$-automorphism $\sigma$ of $R$ such that $\sigma(a)=\gamma(a)$ for all $a \in A, \sigma(y)=\rho y$ and $\sigma(x)=\rho^{-1} x$.

1.4. Quantized Weyl algebras. The quantized Weyl algebra $A_{n}^{\bar{q}, \Lambda}$ in $2 n$ variables $y_{1}, x_{1}, \ldots, y_{n}, x_{n}$ studied in [1], [3], [5], [9] is obtained from the base field $k$ by $n$ applications of the construction in 1.3. The first choice of $v$ is 1 and, at each subsequent application, $v$ is the Casimir element from the previous application. 
The relations are as follows: for $1 \leq i<j \leq n$,

$$
\begin{aligned}
x_{i} x_{j} & =q_{i} \lambda_{i j} x_{j} x_{i}, \quad y_{j} y_{i}=\lambda_{j i} y_{i} y_{j}, \\
x_{i} y_{j} & =\lambda_{j i} y_{j} x_{i}, \quad x_{j} y_{i}=q_{i} \lambda_{i j} y_{i} x_{j}, \\
x_{j} y_{j}-q_{j} y_{j} x_{j} & =1+\sum_{i=1}^{j-1}\left(q_{i}-1\right) y_{i} x_{i},
\end{aligned}
$$

where $\Lambda$ is as in 1.2 and $\bar{q}=\left(q_{1}, q_{2}, \ldots, q_{n}\right)$ is an $n$-tuple of elements of $k \backslash\{0,1\}$. Thus each $y_{j}$ (resp. $x_{j}$ ) semicommutes with each $y_{i}$ (resp. $x_{i}$ ) and, provided $i \neq j$, with each $x_{i}$ (resp. $y_{i}$ ).

There are $n$ normal elements

$$
z_{i}=x_{i} y_{i}-y_{i} x_{i}=1+\sum_{j=1}^{i}\left(q_{j}-1\right) y_{j} x_{j}=q_{i}^{-1}\left(1+\sum_{j=1}^{i}\left(q_{j}-1\right) x_{j} y_{j}\right),
$$

which semicommute with each of the generators and commute with each other:

$$
z_{j} y_{i}=\left\{\begin{array}{l}
y_{i} z_{j} \text { if } j<i, \\
q_{i} y_{i} z_{j} \text { if } j \geq i,
\end{array} \quad z_{j} x_{i}=\left\{\begin{array}{l}
x_{i} z_{j} \text { if } j<i, \\
q_{i}^{-1} x_{i} z_{j} \text { if } j \geq i,
\end{array} \quad z_{i} z_{j}=z_{j} z_{i} .\right.\right.
$$

The last of the listed relations for $A_{n}^{\bar{q} . \Lambda}$ can be rewritten

$$
x_{j} y_{j}-q_{j} y_{j} x_{j}=z_{j-1} \text {. }
$$

In the notation of $1.3, A_{n}^{\bar{q}, \Lambda}=R\left(A_{n-1}^{\bar{q}, \Lambda}, \alpha_{n}, z_{n-1}, q_{n}\right)$, where

$$
\alpha_{n}: y_{i} \mapsto \lambda_{n i} y_{i}, \quad x_{i} \mapsto \lambda_{i n} x_{i} .
$$

The automorphism $\gamma=\gamma_{n}$ of $A_{n-1}^{\bar{q} . \Lambda}$ induced by $z_{n-1}$ is given by

$$
\gamma_{n}: y_{i} \mapsto q_{i} y_{i}, \quad x_{i} \mapsto q_{i}^{-1} x_{i} .
$$

There is abuse of notation here in the use of the same superscripts for $A_{n}^{\bar{q} \wedge \Lambda}$ and $A_{n=1}^{\bar{q} . \Lambda}$. The matrices of parameters for $A_{n-1}^{\bar{q}, \Lambda}$ are of course submatrices of $\bar{q}$ and $\Lambda$, so no confusion should arise.

The subalgebra $O$ generated by $y_{1}, y_{2}, \ldots, y_{n}$ is the coordinate ring $A(\Lambda)$ of quantum $n$-space, the $x_{i}$ 's and $z_{i}$ 's act as partial $q_{i}$-difference operators and automorphisms respectively on $\sigma$, see $[9,2.9]$. In particular, when $n=2$, the actions of $x_{1}, x_{2}, y_{1}, y_{2}, z_{1}$ and $z_{2}$ on a typical monomial $y_{1}^{i} y_{2}^{j}$ in $\mathcal{O}$ are as follows:

$$
\begin{aligned}
x_{1}: y_{1}^{i} y_{2}^{j} \mapsto\left(\frac{1-q_{1}^{i}}{1-q_{1}}\right) y_{1}^{i-1} y_{2}^{j}, & x_{2}: y_{1}^{i} y_{2}^{j} \mapsto\left(\frac{1-q_{2}^{j}}{1-q_{2}}\right)\left(q_{1} \lambda_{12} y_{1}\right)^{i} y_{2}^{j-1}, \\
y_{1}: y_{1}^{i} y_{2}^{j} \mapsto y_{1}^{i+1} y_{2}^{j}, & y_{2}: y_{1}^{i} y_{2}^{j} \mapsto\left(\lambda_{21} y_{1}\right)^{i} y_{2}^{j+1}, \\
z_{1}: y_{1}^{i} y_{2}^{j} \mapsto\left(q_{1} y_{1}\right)^{i} y_{2}^{j}, & z_{2}: y_{1}^{i} y_{2}^{j} \mapsto\left(q_{1} y_{1}\right)^{i}\left(q_{2} y_{2}\right)^{j} .
\end{aligned}
$$

1.5. An alternative quantized Weyl algebra. The alternative quantized Weyl algebra $\mathscr{A}_{n}^{\bar{q} . \Lambda}$ is also obtained from the base field $k$ by $n$ applications of the construction in 1.3 but with all choices of $v$ taken to be 1 . In the notation of $1.3, \mathscr{A}_{n}^{\bar{q}, \Lambda}=$ 
$R\left(A_{n-1}^{\bar{q}, \Lambda}, \alpha_{n}, 1, q_{n}\right)$, where the rules for $\alpha_{n}$ are as in 1.4. As $v$ is always $1, \gamma_{n}$ is always the identity automorphism. The resulting relations are as follows: for $1 \leq i<j \leq n$,

$$
\begin{gathered}
x_{i} x_{j}=\lambda_{i j} x_{j} x_{i}, \quad y_{j} y_{i}=\lambda_{j i} y_{i} y_{j}, \\
x_{i} y_{j}=\lambda_{j i} y_{j} x_{i}, \quad x_{j} y_{i}=\lambda_{i j} y_{i} x_{j}, \\
x_{j} y_{j}-q_{j} y_{j} x_{j}=1 .
\end{gathered}
$$

There are again $n$ normal elements but with a more symmetric form

$$
z_{i}=x_{i} y_{i}-y_{i} x_{i}=1+\left(q_{i}-1\right) y_{i} x_{i}=q_{i}^{-1}\left(1+\left(q_{i}-1\right) x_{i} y_{i}\right)
$$

and more symmetric identities

$$
z_{j} y_{i}=\left\{\begin{array}{l}
y_{i} z_{j} \text { if } j \neq i, \\
q_{i} y_{i} z_{j} \text { if } j=i,
\end{array} \quad z_{j} x_{i}=\left\{\begin{array}{l}
x_{i} z_{j} \text { if } j \neq i, \\
q_{i}^{-1} x_{i} z_{j} \text { if } j=i,
\end{array} \quad z_{i} z_{j}=z_{j} z_{i} .\right.\right.
$$

The subalgebra $O$ generated by $y_{1}, y_{2}, \ldots, y_{n}$ is again $A(\Lambda)$ and the $x_{i}$ 's again act as partial $q_{i}$-difference operators on $\mathcal{O}$ :

$$
\begin{aligned}
x_{1}: y_{1}^{i} y_{2}^{j} \mapsto\left(\frac{1-q_{1}^{i}}{1-q_{1}}\right) y_{1}^{i-1} y_{2}^{j}, & x_{2}: y_{1}^{i} y_{2}^{j} \mapsto\left(\frac{1-q_{2}^{j}}{1-q_{2}}\right)\left(\lambda_{12} y_{1}\right)^{i} y_{2}^{j-1}, \\
y_{1}: y_{1}^{i} y_{2}^{j} \mapsto y_{1}^{i+1} y_{2}^{j}, & y_{2}: y_{1}^{i} y_{2}^{j} \mapsto\left(\lambda_{21} y_{1}\right)^{i} y_{2}^{j+1}, \\
z_{1}: y_{1}^{i} y_{2}^{j} \mapsto\left(q_{1} y_{1}\right)^{i} y_{2}^{j}, & z_{2}: y_{1}^{i} y_{2}^{j} \mapsto y_{1}^{i} \mapsto y_{1}^{i}\left(q_{2} y_{2}\right)^{j} .
\end{aligned}
$$

In comparison with the actions given above for $A_{2}^{\bar{q}, \wedge}$, the only changes are in the scalar appearing in the action of $x_{2}$ and in the automorphism for $z_{2}$ which shows more symmetry.

1.6. Completely prime ideals. We list here two results which will be used to establish that certain ideals are completely prime. The first is well known; see for example $[6,2.1(v i)]$. Given the restriction in 1.3 that $v \neq 0$, the second is a special case of $[9,2.7]$.

Lemma. (i) Let $R$ be a ring with an automorphism $\alpha$ and an $\alpha$-derivation $\delta$ and let $S=R[x ; \alpha, \delta]$. Let $I$ be an ideal of $R$ such that $\alpha(I)=I$ and $\delta(I) \subseteq I$. Then IS is an ideal of $S$ and there are an induced automorphism, also denoted $\alpha$, of $R / I$ and an induced $\alpha$-derivation, also denoted $\delta$, of $R / I$. There is an isomorphism $\theta:(R / I)[x ; \alpha, \delta] \rightarrow S / I S$ with $\theta(x)=x$ and $\theta(r+I)=r+I S$.

(ii) In the notation of 1.3 , if $A$ is a domain and $R=R(A, \alpha, v, \rho)$ then $z R$ is a completely prime ideal of $R$.

1.7. Localizations. In [9] it is shown that, for $1 \leq i \leq n$, the set $\left\{y_{i}^{\prime}\right\}_{j \geq 1}$ is a right and left Ore set in $A_{n}^{\bar{q}, \Lambda}$. The same is true, for the same reasons, in $\mathscr{A}_{n}^{\bar{q}, \Lambda}$.

We denote by $B_{n}^{\bar{q}, \Lambda}$ the localization of $A_{n}^{\bar{q}, \Lambda}$ obtained by inverting the $n$ normal elements $z_{i}, 1 \leq i \leq n$. It is shown in [9] that, provided no $q_{i}$ is a root of unity, $B_{n}^{\bar{q}, \Lambda}$ is simple with Krull and global dimension $n$. We denote the corresponding localization of $\mathscr{A}_{n}^{\bar{q}, \Lambda}$ by $\mathscr{B}_{n}^{\bar{q}, \Lambda}$. The methods of [9] can be adapted to show that, provided no $q_{i}$ is a root of unity, $\mathscr{B}_{n}^{\bar{q}, \Lambda}$ is simple with Krull and global dimension $n$. Alternatively, this follows 
from the corresponding result for $B_{n}^{\bar{q} \wedge}$ because there is an isomorphism $\theta: \mathscr{B}_{n}^{\bar{q} \Lambda} \rightarrow B_{n}^{\bar{q}, \Lambda}$ with $\theta: y_{i} \mapsto y_{i} ; x_{i} \mapsto z_{i-1}^{-1} x_{i}$, where $z_{0}=1$.

1.8. Skew commutator formula. The following formula, which holds for $d \geq 1$, is the special case of $[9,2.6(\mathrm{i})]$ appropriate to those rings of the form $R(A, \alpha, v, \rho)$ in 1.3 with $\alpha(v)=v$ and $\rho \neq 1$.

$$
x y^{d}-\rho^{d} y^{d} x=\frac{1-\rho^{d}}{1-\rho} v y^{d-1} .
$$

1.9. The Noetherian condition. All rings considered in this paper are either iterated skew polynomial rings over a field $k$ or are formed from such rings by taking homomorphic images and/or localizations. By well-known results, see [7] or [12], they are all right and left Noetherian.

2. Prime ideals of $A(\Lambda)$. Proposition 2.2 below is very easy to prove. However it establishes patterns for the hypotheses, results and methods in the subsequent sections on quantized Weyl algebras. The method in 2.2 involves localization and the following simplicity criterion for $P(\Lambda)$ due to McConnell and Pettit [11].

2.1. Proposition. If $n \geqslant 2$ then $P(\Lambda)$ is simple if and only if the only integers $t_{i}$, $1 \leq i \leq n$, such that $\prod_{i=1}^{n} \lambda_{i j}^{i_{i j}}=1$ for all $j$ are $t_{i}=0$.

2.2. Proposition. For $1 \leq i \leq n$, let $G_{i}(\Lambda)=\left\langle\lambda_{i j}: 1 \leq j \leq n\right\rangle$, the subgroup of $k^{*}$ generated by the entries on the $i$-th row of $\Lambda$ (or, equivalently, by the entries in the $i$-th column of $\Lambda$ ). Suppose that each $G_{i}(\Lambda)$ has rank $n-1$. Then every nonzero nonmaximal prime ideal of $A(\Lambda)$ is generated by a nonempty proper subset of $\left\{x_{i}: 1 \leq i \leq n\right\}$ and every maximal ideal of $A(\Lambda)$ is generated by $x_{1}, x_{2}, \ldots, x_{i}-\mu, \ldots, x_{n}$ for some $i, 1 \leq i \leq n$, and some $\mu \in k$.

Proof. The result is certainly true when $n=1$ in which case $A(\Lambda)=k\left[x_{1}\right]$. Let $n>1$. Under the stated hypothesis on $G_{i}(\Lambda), 2.1$ applies to show that $P(\Lambda)$ is simple. Thus every nonzero prime ideal of $A(\Lambda)$ must contain one of the normal elements $x_{i}$. It is clear that $A(\Lambda) / x_{i} A(\Lambda) \simeq A\left(\Lambda^{\prime}\right)$ where $\Lambda^{\prime}$ is the $(n-1) \times(n-1)$ matrix obtained from $\Lambda$ by deleting row $i$ and column $i$. Also, each $G_{j}\left(\Lambda^{\prime}\right)$ must have rank $n-2$. The result follows easily by induction.

2.3. Remark. The condition that the rank of each $G_{i}(\Lambda)$ is $n-1$ is significantly weaker than the condition that the rank of the subgroup $G(\Lambda)$ generated by all the entries in $\Lambda$ takes its maximal value, namely $\frac{1}{2} n(n-1)$. It is possible for the rank of each $G_{i}(\Lambda)$ to be $n-1$ but for the rank of $G(\Lambda)$ to be $n-1$ if $n$ is even and $n$ if $n$ is odd. Examples for $n=4$ and 5 are as follows:

$$
\left[\begin{array}{cccc}
1 & a & b & c \\
a^{-1} & 1 & c & b \\
b^{-1} & c^{-1} & 1 & a \\
c^{-1} & b^{-1} & a^{-1} & 1
\end{array}\right],\left[\begin{array}{ccccc}
1 & a & b & c & d \\
a^{-1} & 1 & c & d & e \\
b^{-1} & c^{-1} & 1 & e & a \\
c^{-1} & d^{-1} & e^{-1} & 1 & b \\
d^{-1} & e^{-1} & a^{-1} & b^{-1} & 1
\end{array}\right] .
$$

Here $a, b, c, d$ and $e$ are elements of $k^{*}$ generating a subgroup of rank 5 . 
For arbitrary $n$, an example can be constructed using an edge-colouring $\mathscr{C}$ of the complete graph $K_{n}$ on $n$ vertices with a minimal number of colours. This number, $m_{n}$, say, is $n-1$ if $n$ is odd, see [2, p. 82]. Let $c_{i}, 1 \leq i \leq m_{n}$, be the colours in $\mathscr{C}$ and let $a_{i}$, $1 \leq i \leq m_{n}$, be elements of $k^{*}$ generating a subgroup of rank $m_{n}$. Let $\Lambda$ be the $n \times n$ matrix satisfying the rules: each $\lambda_{i i}=1$ and, whenever $j>i, \lambda_{i j}^{-1}=\lambda_{j i}=a_{r}$ where $c_{r}$ is the colour of the edge joining vertices $i$ and $j$. Then, as no two edges of the same colour meet at a vertex, none of the $a_{i}$ 's can appear twice in any column. Thus each $G_{i}(\Lambda)$ has rank $n-1$. We thank Victor Bryant for pointing out the connection with edge-colourings.

\section{Prime ideals of $\mathscr{A}_{n}^{\bar{q}, \Lambda}$.}

3.1. Notation. For $\mathscr{A}_{n}^{\bar{q}, \Lambda}$, the analogous condition on the parameters to that in 2.2 involves the subgroups $H_{i}=H_{i}(\Lambda, \bar{q})$ generated by $G_{i}$ and $q_{i}$. The maximal rank for each of these is $n$ whereas the maximal rank of the group $H=H(\Lambda, \bar{q})$ generated by all the parameters $\lambda_{i j}$ and $q_{i}$ is $\frac{1}{2} n(n+1)$. We shall show that if $n>1$ and each $H_{i}(\Lambda, \bar{q})$ has rank $n$ then every nonzero prime ideal of $\mathscr{A}_{n}^{\bar{q}, \Lambda}$ is generated by a subset of the set $\left\{z_{i}: 1 \leq i \leq n\right\}$ of normal elements. When considering such a subset $Z$, the symmetry in the defining relations for $\mathscr{A}_{n}^{\bar{q}, \Lambda}$ allows us to assume, without loss of generality, that $Z=\left\{z_{1}, z_{2}, \ldots, z_{m}\right\}$. Throughout this section, we fix $\Lambda$ and $\bar{q}$ and write $\mathscr{A}_{n}$ for $\mathscr{A}_{n}^{\bar{q}, \Lambda}$. We let $P_{m}$ denote the ideal $z_{1} \mathscr{A}_{n}+z_{2} \mathscr{A}_{n}+\ldots+z_{m} \mathscr{A}_{n}$ of $\mathscr{A}_{n}$.

It is cumbersome to use the standard overlining notation for images in factor rings of $A_{n}$ and $\mathscr{A}_{n}$ so we shall often abuse notation and write, for example, $y_{i}$ for the image of $y_{i}$ in a factor ring. We shall occasionally indicate such abuses by inserting the phrase "the images of" in brackets.

\subsection{Lemma. For $1 \leq m \leq n, P_{m}$ is a completely prime ideal of $\mathscr{A}_{n}$.}

Proof. We first consider $P_{n}$. For $1 \leq i \leq n, 1+\left(q_{i}-1\right) y_{i} x_{i}=z_{i} \in P_{n}$ and $1+$ $\left(q_{i}-1\right) x_{i} y_{i}=q_{i} z_{i} \in P_{n}$ so, modulo $P_{n}$, each $y_{i}$ is invertible with inverse $\left(1-q_{i}\right) x_{i}$. Consequently, $\mathscr{A}_{n} / P_{n}$ is isomorphic to $P(\Lambda)$ which is certainly a domain. For $1 \leq m<n$, the elements $z_{1}, z_{2}, \ldots, z_{m}$ generate a completely prime ideal of $\mathscr{A}_{m}$ and they are fixed (resp. annihilated) by each of the automorphisms (resp. derivations) used in the construction of $\mathscr{A}_{n}$ from $\mathscr{A}_{m}$. It follows from $1.6(\mathrm{i})$ that $P_{m}$ is completely prime.

3.3. Proposition. Suppose that $1 \leq m \leq n$ and let $T=\mathscr{A}_{n} / P_{m}$. If each of the groups $H_{i}$ has rank $n$ then the localization $T_{1}$ of $T$ at the multiplicatively closed set generated by the images in $T$ of the normal elements $z_{m+1}, z_{m+2}, \ldots, z_{n}$ is simple.

Proof. Let $T=\mathscr{A}_{n} / P_{m}$. Each of the sets $\left\{y_{i}^{j}\right\}_{j \geq 1}$ and $\left\{z_{i}^{j}\right\}_{j \geq 1}$ is a right and left Ore set in $\mathscr{A}_{n}$, see 1.7 , and the $2 n$ elements $y_{i}, z_{j}$ semicommute with each other. It follows that the multiplicatively closed set generated by these elements $y_{i}, z_{i}, 1 \leq i \leq n$, is a right and left Ore set in $\mathscr{A}_{n}$. The image $\mathscr{C}$ in $T$ of this Ore set is a right and left denominator set in $T$. Let $T_{2}$ denote the localization of $T$ at $\mathscr{C}$. For $1 \leq i \leq m$, the image of $y_{i}$ is already invertible in $T$ and the image of $z_{i}$ is zero. Thus $T_{2}$ is obtained from $T$ by inverting the 
images of $y_{i}$ and $z_{i}$ for $m+1 \leq i \leq n$. It is generated, as a $k$-algebra, by (the images of) $y_{1}^{ \pm 1}, y_{2}^{ \pm 1}, \ldots, y_{n}^{ \pm 1}, z_{m+1}^{ \pm 1}, z_{m+2}^{ \pm 1}, \ldots$ and $z_{n}^{ \pm 1}$ subject to the relations

$$
y_{j} y_{i}=\lambda_{j i} y_{i} y_{j}, \quad z_{j} y_{i}=\left\{\begin{array}{l}
y_{i} z_{j} \text { if } j \neq i, \\
q_{i} y_{i} z_{j} \text { if } j=i
\end{array} .\right.
$$

Thus $T_{2} \simeq P\left(\Lambda^{\dagger}\right)$ where $\Lambda^{\dagger}$ is a $(2 n-m) \times(2 n-m)$ matrix of the form

$$
\left[\begin{array}{cc}
\Lambda & \Gamma \\
\Gamma^{*} & \Theta
\end{array}\right]
$$

Here $\Gamma$ is $n \times(n-m)$ with all entries equal to 1 except that, for $m+1 \leq i \leq n$, the $(i, n+1)$-entry is $q_{i}^{-1}, \Gamma^{*}$ is obtained from $\Gamma$ by inverting all the entries and taking the transpose and $\Theta$ is $(n-m) \times(n-m)$ with all entries equal to 1 . As each $H_{i}$ has maximal rank, it follows from 2.1 that $T_{2}$ is simple. For $0 \leq j \leq n-m$, let $U_{j}$ be the localization of $T_{1}$ obtained by inverting $y_{m+1}, y_{m+2}, \ldots, y_{m+j}$. Thus $U_{0}=T_{1}$ and $U_{n-m}=T_{2}$ is simple. Let $j>0$ and suppose that $U_{j}$ is simple. Let $I$ be a nonzero ideal of $U_{j-1}$ and let $y=y_{m+j}$, $x=x_{m+j}$ and $q=q_{m+j}$. By the simplicity of $U_{j}$, which is obtained from $U_{j-1}$ by inverting the powers of $y, y^{d} \in I$ for some $d \geq 0$ and we choose the minimal such $d$. The formula 1.8 gives

$$
x y^{d}-q^{d} y^{d} x=\frac{\left(1-q^{d}\right)}{(1-q)} y^{d-1} .
$$

As $q$ cannot be a root of unity, it follows that $y^{d-1} \in I$, contradicting the minimality of $d$ unless $d=0$. Thus $1 \in I$ and so $U_{j-1}$ is simple. By induction, each $U_{i}$ is simple and in particular $U_{0}=T_{1}$ is simple.

3.4. Proposition. If $n>1$ and each of the groups $H_{i}(\Lambda, \bar{q})$ has rank $n$ then every nonzero prime ideal $P$ of $\mathscr{A}_{n}^{\bar{q}, \Lambda}$ is generated by a non-empty subset of the set of normal elements $\left\{z_{i}: 1 \leq i \leq n\right\}$.

Proof. In 1.7, we observed that the localization $\mathscr{B}_{n}^{\bar{q}, \Lambda}$ is simple. Hence $z_{i} \in P$ for some i. By symmetry, we can renumber so that, for some $m, z_{1}, z_{2}, \ldots, z_{m} \in P$ and $z_{m+1}$, $z_{m+2}, \ldots, z_{n} \notin P$. Thus $P_{m} \subseteq P$. By 3.3 , the localization of $\mathscr{A}_{n} / P_{m}$ obtained by inverting the (images of) the normal elements $z_{m+1}, z_{m+2}, \ldots, z_{n}$ is simple so if $P \neq P_{m}$ then $P$ must contain $z_{j}$ for some $j$ with $m<j \leq n$, contradicting the choice of $m$. Thus $P=P_{m}$ and, undoing the reordering, $P$ is generated by a non-empty subset of $\left\{z_{i}: 1 \leq i \leq n\right\}$.

3.5. COROLlaRY. Under the hypotheses of 3.4, every prime ideal of $\mathscr{A}_{n}^{\bar{q}, \Lambda}$ is (right and left) localizable.

Proof. As the prime ideals are all generated by normal elements they have the (right and left) Artin-Rees property, see [12, Theorem 4.2.6]. By Noetherian induction all ideals have the Artin-Rees property and the result follows from [12, Theorem 4.2.11(i)].

We shall see in $4.13(v)$ that the analogous result for $A_{n}^{\bar{q}, \Lambda}$ is false.

4. Prime ideals of $A_{n}^{\bar{q}, \Lambda}$. Throughout this section, we fix $\Lambda$ and $\bar{q}$ and write $A_{n}$ for $A_{n}^{\bar{q}, \Lambda}$. The prime spectrum of $A_{n}$ is more complex than that of $\mathscr{A}_{n}^{\bar{q} . \Lambda}$. Considerations of symmetry, as in Section 3, do not apply to $A_{n}^{\bar{q}, \Lambda}$. Moreover, the ideals analogous to those 
in 3.2 need not be prime. For example, the ideal generated by the normal elements $z_{1}$ and $z_{2}$ is not prime.

4.1. Lemma. Let $I$ be an ideal of $A_{n}$ and let $1 \leq i \leq n$.

(i) If $y_{i} \in I$ or $x_{i} \in I$ then $z_{i} \in I$ and, provided $i>1, z_{i-1} \in I$.

(ii) If $i>1, z_{i-1} \in I$ and $z_{i} \in I$ then, modulo $I, x_{i} y_{i}=0=y_{i} x_{i}$.

(iii) If $z_{i} \in I$ or if $i>1$ and $z_{i-1} \in I$ then $x_{i}$ and $y_{i}$ are normal modulo $I$.

(iv) Suppose that $I$ is prime and that $i>1$. If $z_{i} \in I$ and $z_{i-1} \in I$ then $y_{i} \in I$ or $x_{i} \in I$.

Proof. (i) and (ii) are consequences of the identities

$$
\begin{aligned}
x_{i} y_{i}-q_{i} y_{i} x_{i} & =z_{i-1}, \\
z_{i}=x_{i} y_{i}-y_{i} x_{i} & =z_{i-1}+\left(q_{i}-1\right) y_{i} x_{i}, \\
q_{i} z_{i} & =z_{i-1}+\left(q_{i}-1\right) x_{i} y_{i} .
\end{aligned}
$$

These also show that if $z_{i} \in I$ then $x_{i}$ and $y_{i}$ commute modulo $I$ and if $z_{i-1} \in I$ then $x_{i}$ and $y_{i}$ semicommute modulo $I$. (iii) follows because $y_{i}$ (resp. $x_{i}$ ) semicommutes with each of the generators of $A_{n}$ except $x_{i}$ (resp. $y_{i}$ ). Finally, (iv) is immediate from (ii) and (iii).

We shall see that every nonzero prime ideal of $A_{n}$ has a normalizing sequence of generators, that, as was the case in Sections 2 and 3, there are only finitely many nonmaximal primes and that, as was the case in Section 2 but not in Section 3, there are infinitely many maximal ideals. We begin with a definition which describes the normalizing sequences for the nonmaximal primes.

4.2. Definition. For a subsequence $S$ of the sequence

$$
N:=\left\{z_{1}, y_{1}, x_{1}, z_{2}, y_{2}, x_{2}, \ldots, z_{n}, y_{n}, x_{n}\right\}
$$

and an integer $i$ with $1 \leq i \leq n$, we say that $i$ features in $S$ if at least one of $z_{i}, y_{i}, x_{i}$ is in $S$. By a $p$-sequence, we mean a subsequence $S$, possibly empty, of $N$ with the following properties:

(i) if $z_{i} \in S$ then $i-1$ does not feature in $S$;

(ii) if $y_{i} \in S$ or $x_{i} \in S$ then $i-1$ does feature in $S$.

Thus a p-sequence is the concatenation of a set of subsequences $S_{i}$ such that the set of subscripts featuring in $S_{i}$ is consecutive, each $S_{i}$ begins with $z_{r_{i}}$ but contains no other $z_{j}$ and there is a gap between the largest subscript featuring in $S_{i}$ and the smallest one in $S_{i+1}$. An example is $\left\{z_{2}, x_{3}, y_{4}, x_{4}, z_{6}, y_{7}\right\}$. The number of terms in a p-sequence $S$ will be called the length of $S$ and will be written len $(S)$.

4.3. Lemma. Let $S$ be a $p$-sequence and let $P$ be the right ideal of $A_{n}$ generated by $S$.

(i) For $1 \leq i \leq n$, if $i$ features in $S$ then $z_{i} \in P$.

(ii) $P$ is an ideal of $A_{n}$ and $S$ is a normalizing sequence of generators for $P$.

Proof. If $i$ features in $S$ then there exist integers $j \geq 1$ and $m \geq 0$ such that $j-1$ does not feature in $S$ (or $j=1), j, j+1, \ldots, j+m$ feature in $S$ and $i=j+m$. Thus $z_{j} \in S$ and, for $j<s \leq j+m, x_{s} \in S$ or $y_{s} \in S$. As $z_{s}=z_{s-1}+\left(q_{s}-1\right) y_{s} x_{s}$ and $q_{s} z_{s}=z_{s-1}+\left(q_{s}-1\right) x_{s} y_{s}$, it follows that each $z_{s} \in P$, in particular $z_{i} \in P$. Thus (i) holds. (ii) is immediate from (i), 4.1(ii) and the normality of the elements $z_{i}$. 
4.4. Inductive methods. Suppose that $n>1$ and let $S^{\prime}=S \cap A_{n-1}$ which is a p-sequence in $A_{n-1}$ and hence, by 4.3 (ii) is a normalizing sequence in $A_{n-1}$. Let $Q$ be the ideal of $A_{n-1}$ generated by $S^{\prime}$ and let $B=A_{n-1} / Q$. The ring $A_{n}$ has the form $R\left(A_{n-1}, \alpha, z_{n-1}, q_{n}\right)=A_{n-1}\left[y_{n} ; \alpha\right]\left[x_{n} ; \beta, \delta\right]$ of 1.3 with $\alpha$ specified in 1.4 . In particular, each $y_{i}, x_{i}$ and $z_{i}$ are eigenvectors for both $\alpha$ and $\beta, \delta\left(A_{n-1}\right)=0$ and $\delta\left(y_{n}\right)=z_{n-1}$. It follows that $Q$ is invariant under each of $\alpha$, the automorphism $\gamma$ induced by $z_{n-1}$, $\beta=\gamma \alpha^{-1}$ and $\delta$. By two applications of 1.6(ii), $Q A_{n}$ is an ideal of $A_{n}$ and, with the convention on induced maps as in $1.6, A_{n} / Q A_{n} \simeq B\left[y_{n} ; \alpha\right]\left[x_{n} ; \beta, \delta\right]$. The next lemma makes explicit the relationship between the rings $A_{n} / P$ and $A_{n-1} / Q$ and is the basis for inductive proofs on the prime ideals of $A_{n}$.

Lemma. Let $S$ be a p-sequence in $A_{n}$. and $x_{n} \notin P$.

(i) If $n$ does not feature in $S$ then $P=Q A_{n}, A_{n} / P=B\left[y_{n} ; \alpha\right]\left[x_{n} ; \beta, \delta\right], z_{n} \notin P, y_{n} \notin P$

(ii) If $x_{n} \in S$ and $y_{n} \notin S$ then $P=Q A_{n}+x_{n} A_{n}, A_{n} / P \simeq B\left[y_{n} ; \alpha\right]$ and $y_{n} \notin P$.

(iii) If $y_{n} \in S$ and $x_{n} \notin S$ then $P=Q A_{n}+y_{n} A_{n}, A_{n} / P=B\left[x_{n} ; \beta\right]$ and $x_{n} \notin P$.

(iv) If $y_{n} \in S$ and $x_{n} \in S$ then $P=Q A_{n}+y_{n} A_{n}+x_{n} A_{n}$ and $A_{n} / P=B$.

(v) If $z_{n} \in S$ then $P=Q A_{n}+z_{n} A_{n}, y_{n} \notin P$ and $x_{n} \notin P$ and $A_{n} / P \simeq R / z_{n} R$, where, in the notation of $1.3, R=R\left(B, \alpha, z_{n-1}, q_{n}\right)$.

Proof. The relationships between $P$ and $Q$ are immediate from the definitions and (i)-(iv) follow easily using the isomorphism $A_{n} / Q A_{n} \simeq B\left[y_{n} ; \alpha\right]\left[x_{n} ; \beta, \delta\right]$. For (v), suppose that $z_{n} \in S$. Then, by definition of p-sequence, $n-1$ cannot feature in $S^{\prime}$. By (i), $z_{n-1} \notin Q$. Thus (the image of) $z_{n-1}$ is a nonzero normal element in $B$ and $A_{n} / Q A_{n}=$ $B\left[y_{n} ; \alpha\right]\left[x_{n} ; \beta, \delta\right]=R\left(B, \alpha, z_{n-1}, q_{n}\right)=R$. Hence $A_{n} / P=A_{n} /\left(Q A_{n}+z_{n} A_{n}\right)=R / z_{n} R$. Viewed as a polynomial in $x_{n}$ over $B\left[y_{n} ; \alpha\right], z_{n}$ has degree 1 and a noninvertible leading coefficient $\left(q_{i}-1\right) y_{n}$. Hence $x_{n} \notin z_{n} R$ and, similarly, $y_{n} \notin z_{n} R$. Applying the isomorphism $R / z_{n} R=A_{n} / P, y_{n} \notin P$ and $x_{n} \notin P$.

4.5. Proposition. Let $S$ be a p-sequence in $A_{n}$ and let $P$ be the ideal of $A_{n}$ generated by $S$. Then $P$ is completely prime.

Proof. This is certainly true when $n=1$, in which case $\left\{z_{1}\right\}$ is the only nonempty p-sequence and $z_{1} A_{1}$ is a completely prime ideal, with factor isomorphic to $k\left[y_{1}^{ \pm 1}\right]$. Inductively, we may assume that if $S^{\prime}=S \cap A_{n-1}$ and $Q=S^{\prime} A_{n-1}$ then $B=A_{n-1} / Q$ is a domain. In each of the cases (i)-(iv) in 4.4 it is immediate that $A_{n} / P$ is a domain while in $4.4(\mathrm{v}), 1.6(\mathrm{ii})$ applies to give the same conclusion. By the definition of p-sequence these five cases are exhaustive so, by induction, $P$ is always completely prime.

4.6. Lemma. In each of the cases listed in 4.4, $P \cap A_{n-1}=Q$. Hence, for $1 \leq i \leq n$,

(i) $z_{i} \in P$ if and only if $i$ features in $S$,

(ii) $y_{i} \in P$ if and only if $y_{i} \in S$,

(iii) $x_{i} \in P$ if and only if $x_{i} \in S$.

Proof. It is routine to check that $P \cap A_{n-1}=Q$ in each case. Hence we have $P \cap A_{i}=\left(S \cap A_{i}\right) A_{i}$ for $1 \leq i \leq n$. For (i) the "if" part is $4.3(\mathrm{i})$ and for (ii) and (iii) the "if" parts are obvious. The "only if" parts hold for the case $i=n$ by 4.4 and for lower values of $i$ because $P \cap A_{i}=\left(S \cap A_{i}\right) A_{i}$. 
4.7. The maximal p-sequence. Let $S$ be the p-sequence $z_{1}, y_{2}, x_{2}, y_{3}, x_{3}, \ldots, y_{n}, x_{n}$ and let $M=S A_{n}$. By 4.1(i), $z_{i} \in M$ for all $i$. Hence if $T$ is any p-sequence and $P$ is the ideal generated by $T$ then $P \subseteq M$. We shall call $S$ the maximal p-sequence in $A_{n}$. Note that, by $n-1$ applications of $4.4(\mathrm{iv})$ and one of $4.4(\mathrm{v}), A_{n} / M=k\left[y_{1}^{ \pm 1}\right]$. It follows that there are infinitely many maximal ideals of $A_{n}$ of the form $M+\left(y_{1}-\mu\right) A_{n}, \mu \in k^{*}$ and that these are the only prime ideals of $A_{n}$ strictly containing $M$.

4.8. Localizations. We aim to show that, under conditions on the parameters, similar to those in 3.4, every nonzero nonmaximal prime ideal of $A_{n}$ is generated by a p-sequence. Our method is similar to that in Section 3 and involves certain localizations $T_{1}$ and $T_{2}$ of $A_{n} / P$ where $P$ is as in 4.3. The conditions on the parameters will be stronger than in 3.4 in that the subgroup $G(\bar{q})$ of $k^{*}$ generated by the parameters $q_{i}, 1 \leq i \leq n$, should also have rank $n$.

Let $S$ be a p-sequence in $A_{n}$, let $P$ be the prime ideal of $A_{n}$ generated by $S$ and let $T$ be the domain $A_{n} / P$. Let

$$
C(S)=\left\{z_{i}: z_{i} \notin P\right\} \cup\left\{y_{i}: y_{i} \notin P \text { and } z_{i} \in P\right\} \cup\left\{x_{i}: x_{i} \notin P \text { and } z_{i} \in P\right\}
$$

and let $D(S)=C(S) \cup\left\{y_{i}: y_{i} \notin P\right\}$. If $z_{i} \in C(S)$ then, as $z_{i}$ semicommutes with each element of $S$ and is normal in $A_{n}, z_{i}$ is normal modulo $P$. Also, if $y_{i} \in C(S)$ then $y_{i}$ is normal modulo $P$ by 4.1(iii). The same is true of $x_{i}$. Thus each element of $C(S)$ is normal modulo $P$. As $x_{i}$ can only be in $C(S)$ when $z_{i} \in P$, in which case $x_{i}$ commutes with $y_{i}$ modulo $P$, the elements of $C(S)$ all semicommute with each other modulo $P$. Therefore the set of images in $T$ of elements of the form $\mu c_{1}^{j_{1}} c_{2}^{j_{2}} \ldots c_{s}^{j_{s}}, \mu \in k^{*}, c_{i} \in C(S), j_{i} \geq 0$ is a right and left Ore set in $T$. Let $T_{1}$ denote the localization of $T$ at this set. Thus $T_{1}$ is obtained from $T$ by inverting the elements of $C(S)$, each of which is normal modulo $P$.

Now consider $y_{i} \in D(S) \backslash C(S)$. By $1.8,\left\{y_{i}^{j}\right\}_{j \geq 1}$ is a right and left Ore set in $A_{n}$ and so its image is a right and left Ore set in the domain $T$. As with $C(S)$, the elements of $D(S)$ semicommute with each other modulo $P$ and the set of images in $T$ of elements of the form $\mu d_{1}^{j_{1}} d_{2}^{j_{2}} \ldots d_{s}^{j_{s}}, \mu \in k^{*}, d_{i} \in D(S), j_{i} \geq 0$ is a right and left Ore set in $T$. Let $T_{2}$ denote the localization of $T$ at this set. Thus $T_{2}$ is obtained from $T$ by inverting the elements of $D(S)$ and from $T_{1}$ by inverting those elements $y_{i}$ such that $y_{i} \notin P$ and $z_{i} \notin P$.

4.9. Proposition. Suppose that each of the groups $H_{i}(\Lambda, \bar{q})$ and $G(\bar{q})$ has rank $n$. Let $S$ be a p-sequence in $A_{n}$, let $P$ be the prime ideal of $A_{n}$ generated by $S$ and let $T$ be the domain $A_{n} / P$. If $S$ is nonmaximal then the localization $T_{2}$ specified in 4.8 is simple.

Proof. As $T$ is generated by (the images of) those elements $y_{i}$ and $x_{i}$ not belonging to $P$, the localization $T_{2}$ is generated by these elements together with the inverses of the elements in $D(S)$. Let $T_{2}^{\prime}$ be the subalgebra of $T_{2}$ generated by (the image of) $E(S) \cup\left\{e^{-1}: e \in E(S)\right\}$, where

$$
E(S)=\left\{y_{i}: y_{i} \notin P\right\} \cup\left\{z_{i}: z_{i} \notin P\right\} \cup\left\{x_{i}: x_{i} \notin P \text { and } y_{i} \in P\right\} .
$$

Note that if $x_{i} \notin P$ but $y_{i} \in P$ then $z_{i} \in P$ so $x_{i} \in D(S)$ and therefore $T_{2}^{\prime}$ is indeed a subalgebra of $T_{2}$. We claim that $T_{2}=T_{2}^{\prime}$. Clearly (the images of) each $z_{i}$ and $y_{i}$ are in $T_{2}^{\prime}$, as are $z_{i}^{-1}$ if $z_{i} \in D(S), y_{i}^{-1}$ if $y_{i} \in D(S)$ and $x_{i}$ if $x_{i} \notin P$ but $y_{i} \in P$. Suppose that $x_{i} \notin P$ and $y_{i} \notin P$. Then $y_{i}^{-1}, z_{i}, z_{i-1} \in T_{2}^{\prime}$ so $x_{i}=\left(\left(q_{i}-1\right) y_{i}\right)^{-1}\left(z_{i}-z_{i-1}\right) \in T_{2}^{\prime}$. Furthermore, if 
$x_{i} \in D(S)$ then $z_{i} \in P$ and, by 4.1 (iv), $z_{i-1} \notin P$, so $x_{i}=\left(\left(q_{i}-1\right) y_{i}\right)^{-1}\left(-z_{i-1}\right)$ and $x_{i}^{-1}=$ $\left(1-q_{i}\right) z_{i-1}^{-1} y_{i} \in T_{2}^{\prime}$. It follows that $T_{2}=T_{2}^{\prime}$.

Let $S^{\prime}=S \cap A_{n-1}$ and let $Q=S^{\prime} A_{n-1}$ as in 4.4. Note that, by 4.6, $E\left(S^{\prime}\right)=E(S) \cap$ $A_{n-1}$. We rewrite the elements of $E(S)$ as $w_{1}, w_{2}, \ldots, w_{m}$, say, where $w_{1}>w_{2}>\ldots>w_{m}$ in the ordering

$$
y_{1}>z_{1}>x_{1}>y_{2}>z_{2}>x_{2}>\ldots>y_{n}>z_{n}>x_{n} .
$$

Then $E\left(S^{\prime}\right)=\left\{w_{1}, w_{2}, \ldots, w_{r}\right\}$ for some $r \leq m$. Note that $y_{1}$ cannot be in $P$ so $w_{1}=y_{1}$.

The elements of $E(S)$ semicommute according to the rules

$$
\begin{array}{rlrl}
x_{i} x_{j} & =q_{i} \lambda_{i j} x_{j} x_{i}, & & y_{j} y_{i}=\lambda_{j i} y_{i} y_{j}, \\
x_{i} y_{j}=\lambda_{j i} y_{j} x_{i}, & x_{j} y_{i}=q_{i} \lambda_{i j} y_{i} x_{j}(j>i)
\end{array}
$$

and

$$
z_{j} y_{i}=\left\{\begin{array}{l}
y_{i} z_{j} \text { if } j<i, \\
q_{i} y_{i} z_{j} \text { if } j \geq i,
\end{array} \quad z_{j} x_{i}=\left\{\begin{array}{l}
x_{i} z_{j} \text { if } j<i, \\
q_{i}^{-1} x_{i} z_{j} \text { if } j \geq i,
\end{array} \quad z_{i} z_{j}=z_{j} z_{i}\right.\right.
$$

from 1.4. Note that $y_{i}$ and $x_{i}$ cannot both appear in $E(S)$.

Let $\Delta=\left[d_{i j}\right]$ be the $m \times m$ matrix determined by the rule

$$
w_{j} w_{i}=d_{j i} w_{i} w_{j}
$$

and let $\Delta^{\dagger}$ be the corresponding $r \times r$ matrix for $E\left(S^{\prime}\right)$. We claim that the algebra $P(\Delta)$ is simple. As $T_{2}=T_{2}^{\prime}$ is a homomorphic image of $P(\Delta)$, it will follow that $T_{2}$ is simple. We shall establish the simplicity of $P(\Delta)$ by induction on $n$. When $n=1$ the only nonmaximal p-sequence $S$ is empty, $E(S)=\left\{y_{1}, z_{1}\right\}$ and

$$
\Delta=\left[\begin{array}{cc}
1 & q_{1}^{-1} \\
q_{1} & 1
\end{array}\right] \text {. }
$$

In this case, $P(\Delta)$ is certainly simple.

Now suppose that $n>1$. Note that the condition on the parameters must hold in $A_{n-1}$ otherwise it would fail in $A_{n}$. By induction, we can assume that either $P\left(\Delta^{\dagger}\right)$ is simple or that $S^{\prime}$ is the maximal p-sequence in $A_{n-1}$, in which case $r=1$ and $\Delta^{\dagger}=$ [1]. With a view to applying 2.1 , let $t_{i}, 1 \leq i \leq m$, be integers such that $\prod_{i=1}^{m} d_{i j}^{t_{i}}=1$ for all $j$. We consider separately the cases (iv) - (v) of 4.4 .

(i) If $n$ does not feature in $S$ then $E(S)=E\left(S^{\prime}\right) \cup\left\{y_{n}, z_{n}\right\}, m=r+2$ and

$$
\Delta=\left[\begin{array}{ll}
\Delta^{\dagger} & \Gamma \\
\Gamma^{*} & \Omega
\end{array}\right], \text { where } \Omega=\left[\begin{array}{cc}
1 & q_{n}^{-1} \\
q_{n} & 1
\end{array}\right],
$$

$\Gamma$ is $r \times 2$ and $\Gamma^{*}$ is obtained from $\Gamma$ by inverting the entries and taking transpose. Each of the entries $d_{i, m-1}, 1 \leq i \leq r$, arises from the semicommutation of $y_{n}$ with either $y_{i}, z_{i}$ or $x_{i}$ for some $i<n$ and so is one of $\lambda_{i n}, 1, \lambda_{n i}, i<n$. Also $d_{m-1, m-1}=1$ and $d_{m, m-1}=q_{n}$. As $H_{n}$ has maximal rank, it follows that $t_{m}=0$. Each of the entries $d_{i m}, 1 \leq i \leq r$, arises from the semicommutation of $z_{n}$ with either $y_{i}, z_{i}$ or $x_{i}$ for some $i<n$ and is one of $q_{i}^{-1}, 1, q_{i}, i<n$. As $d_{m-1, m}=q_{n}^{-1}, d_{m m}=1$ and $G(\bar{q})$ has maximal rank, it follows that $t_{m-1}=0$. Hence 
$\prod_{i=1}^{r} d_{i j}^{t_{i}}=1$ for each $j, 1 \leq j \leq r$. In the case where $S^{\prime}$ is nonmaximal it follows from the simplicity of $P\left(\Delta^{\dagger}\right)$ that each $t_{j}=0$ and hence that $P(\Delta)$ is simple. When $S^{\prime}$ is maximal, $m=3, E(S)=\left\{y_{1}, y_{n}, z_{n}\right\}$ and

$$
\Delta=\left[\begin{array}{ccc}
1 & \lambda_{1 n} & q_{1}^{-1} \\
\lambda_{n 1} & 1 & q_{n}^{-1} \\
q_{1} & q_{n} & 1
\end{array}\right] .
$$

The maximality of the rank of $G(\bar{q})$ shows that $t_{1}=t_{2}=0=t_{3}$ and hence that $P(\Delta)$ is again simple.

(ii) If $x_{n} \in S$ and $y_{n} \notin S$ then $E(S)=E\left(S^{\prime}\right) \cup\left\{y_{n}\right\}, m=r+1$ and

$$
\Delta=\left[\begin{array}{cc}
\Delta^{\dagger} & \Gamma \\
\Gamma^{*} & 1
\end{array}\right]
$$

where $\Gamma$ is $r \times 1$ and $\Gamma^{*}$ is obtained from $\Gamma$ by inverting the entries and taking transpose. Here $d_{m 1}=\lambda_{n 1}$. Each entry $d_{i 1}, 1 \leq i \leq r$, arises from the semicommutation of $y_{1}$ with either $y_{i}, z_{i}$ or $x_{i}$ for some $i<n$ and is one of $\lambda_{i 1}, q_{1}, q_{1} \lambda_{1 i}, i<n$. As $H_{1}$ has maximal rank, it follows that $t_{m}=0$. In the case where $S^{\prime}$ is nonmaximal it follows, as in (i), that $P(\Delta)$ is simple. When $S^{\prime}$ is maximal, $m=2, E(S)=\left\{y_{1}, y_{n}\right\}$,

$$
\Delta=\left[\begin{array}{cc}
1 & \lambda_{1 n} \\
\lambda_{n 1} & 1
\end{array}\right]
$$

and it is easy to check that $P(\Delta)$ is again simple.

(iii) If $y_{n} \in S$ and $x_{n} \notin S$ then $E(S)=E\left(S^{\prime}\right) \cup\left\{x_{n}\right\}, m=r+1$. In this case $P(\Delta)$ is again simple, the details being much as in (ii) but with $d_{m 1}=q_{1} \lambda_{1 n}$.

(iv) If $y_{n} \in S$ and $x_{n} \in S$ then $E(S)=E\left(S^{\prime}\right), m=r$ and $P(\Delta)$ is simple except in the case where $S^{\prime}$ is the maximal p-sequence in $A_{n-1}$. In the exceptional case, $S=S^{\prime} \cup\left\{y_{n}, x_{n}\right\}$ is the maximal p-sequence in $A_{n}$.

(v) If $z_{n} \in S$ then again $E(S)=E\left(S^{\prime}\right) \cup\left\{y_{n}\right\}, m=r+1$ and, as in (ii) $P(\Delta)$ is simple.

This completes the inductive proof that, provided $S$ is nonmaximal, $P(\Delta)$ is simple. It follows that $T_{2} \approx P(\Delta)$ is simple.

4.10. Proposition. Under the hypotheses of 4.9 , if $S$ is nonmaximal then the localization $T_{1}$ specified in 4.8 is simple.

Proof. The ring $T_{2}$ is obtained from $T_{1}$ by inverting those elements $y_{i}$ such that $y_{i} \notin P$ and $z_{i} \notin P$. List these as $y_{i_{1}}, y_{i_{2}}, \ldots, y_{i_{s}}$, say. Note that, by 4.1(i) and 4.1(iv), $x_{i_{j}} \notin P$ and $z_{i_{j}-1} \notin P$ for $1 \leq j \leq s$. Thus each $z_{i_{j}-1}$ is invertible in $T_{1}$.

The proof that $T_{1}$ is simple is similar to the last step in the proof of 3.3, with $y_{m+1}$, $y_{m+2}, \ldots, y_{n}$ replaced by $y_{i_{1}}, y_{i_{2}}, \ldots, y_{i_{s}}$. Thus, if $U_{j}$ is the localization of $T_{1}$ obtained by inverting $y_{i_{1}}, y_{i_{2}}, \ldots, y_{i_{j}}$ then $U_{0}=T_{1}, U_{s}=T_{2}$ is simple by 4.9 and 1.8 can be applied to show that each $U_{i}$ is simple and in particular that $U_{0}=T_{1}$ is simple. The appropriate case of 1.8 is

$$
x y^{d}-q^{d} y^{d} x=\frac{\left(1-q^{d}\right)}{(1-q)} z_{i_{j}-1} y^{d-1}
$$


where $y=y_{i_{i}}, x=x_{i_{i}}$ and $q=q_{i_{i}}$. As $q$ cannot be a root of unity and $z_{i_{j}-1}$ is invertible in $T_{1}$, this can be applied in the same way as the corresponding formula in 3.3 .

4.11. Proposition. Under the hypotheses of 4.9 , let $S$ be a nonmaximal p-sequence and let $P$ be the prime ideal of $A_{n}$ generated by $S$. Let $P^{\prime}$ be a prime ideal of $A_{n}$ strictly containing $P$. Then there exists a p-sequence $S^{\prime}$ with $\operatorname{len} S^{\prime}=\operatorname{len} S+1$ and with $P \subset S^{\prime} A_{n} \subseteq P^{\prime}$.

Proof. By 4.10, the localization $T_{1}$ of $A_{n} / P$ is simple. This localization is obtained by inverting the elements of $C(S)$ which are all normal. Hence $P^{\prime}$ contains an element $r$ of one of the forms $z_{i}$, where $z_{i} \notin P, y_{i}$, where $y_{i} \notin P$ and $z_{i} \in P$ or $x_{i}$, where $x_{i} \notin P$ and $z_{i} \in P$.

Suppose that $r=z_{i}$. Then $i$ does not feature in $S$ by 4.3(i). A p-sequence $S^{\prime}$ with the required properties can be obtained in one of the following ways.

(i) If neither $i-1$ nor $i+1$ features in $S$ then insert $z_{i}$ in $S$.

(ii) If $i-1$ features in $S$ but $i+1$ does not then $z_{i-1} \in S$ and, by 4.1(iv), $P^{\prime}$ contains either $x_{i}$ or $y_{i}$. Insert either $x_{i}$ or $y_{i}$ in $S$.

(iii) If $i+1$ features in $S$ but $i-1$ does not then $z_{i+1} \in S$ and, by 4.1(iv), $P^{\prime}$ contains either $x_{i+1}$ or $y_{i+1}$. Replace $z_{i+1}$ by $z_{i}, y_{i+1}$ or by $z_{i}, x_{i+1}$ as appropriate.

(iv) If $i-1$ and $i+1$ both feature in $S$ then, by 4.1 (iv), $P^{\prime}$ contains either $x_{i+1}$ or $y_{i+1}$ and either $x_{i}$ or $y_{i}$. Replace $z_{i+1}$ by $x_{i}, x_{i+1}$, by $y_{i}, x_{i+1}$, by $x_{i}, y_{i+1}$ or by $y_{i}, y_{i+1}$ as appropriate.

Now suppose that $r=y_{i}$ or $r=x_{i}$ where $z_{i} \in P$. Thus $i$ features in $S$ by 4.6(i) and $z_{i-1} \in P^{\prime}$ by 4.1(i). A p-sequence $S^{\prime}$ with the required properties can be obtained in one of the following ways.

(i) If neither $i-1$ nor $i-2$ features in $S$ then $z_{i} \in S$. Replace it by $z_{i-1}, r$.

(ii) If $i-2$ features in $S$ but $i-1$ does not then $z_{i} \in S$ and, by 4.1(iv), $P^{\prime}$ contains either $x_{i-1}$ or $y_{i-1}$. Replace $z_{i}$ by $x_{i-1}, r$ or by $y_{i-1}, r$ as appropriate.

(iii) If $i-1$ features in $S$ then insert $r$ in $S$.

4.12. Proposition. Suppose that each of the groups $H_{i}(\Lambda, \bar{q})$ and $G(\bar{q})$ has rank $n$. Every nonmaximal prime ideal of $A_{n}$ is generated by a p-sequence and every maximal ideal of $A_{n}$ has the form $M+\left(y_{1}-\mu\right) A_{n}$ where $M$ is generated by the maximal $p$-sequence in $A_{n}$ and $\mu \in k^{*}$.

Proof. Let $P^{\prime}$ be a prime ideal of $A_{n}$. Let $S$ be a p-sequence of maximal length contained in $P^{\prime}$ and let $P$ be the prime ideal of $A_{n}$ generated by $S$. Thus $P \subseteq P^{\prime}$. If $S$ is not the maximal p-sequence then by 4.11 and the maximality of len $(S), P^{\prime}=P$. On the other hand, if $S$ is the maximal p-sequence then, by 4.8 , either $P=M$ or $P=M+\left(y_{1}-\mu\right) A_{n}$ for some $\mu \in k^{*}$.

4.13. Remarks. (i) Note that, unlike the situation in Section 3, the spectrum in the case $n=1$ is typical of the general case.

(ii) Under the hypotheses of 4.12 , the maximal ideals of $A_{n}$ have height $2 n$, whereas, in 3.4, those of $\mathscr{A}_{n}$ have height $n$. In $A_{n}$ the ideal generated by the maximal p-sequence is the unique prime of height $2 n-1$ and contains all the nonmaximal primes. In $\mathscr{A}_{n}$, there 
are $2^{n}$ prime ideals including a unique maximal ideal. Rigal $[14,3.16]$ computes the number of nonmaximal primes in $A_{n}$ which is $\frac{1}{2}\left((2+\sqrt{ } 2)^{n}+(2-\sqrt{ } 2)^{n}\right)$.

(iii) Let $P_{0} \subset P_{1} \subset \ldots \subset P_{m}$ be a saturated chain of prime ideals of $A_{n}$. Under the hypotheses of $4.12, P_{0}$ is generated by a p-sequence of length $l$, say, and $P_{m}$ is generated either by a p-sequence of length $h$, say, or by the maximal p-sequence together with $y_{1}-\mu$ for some $\mu$, in which case we set $h=2 n$. In the latter case, $P_{m-1}$ must be generated by the maximal p-sequence, which has length $2 n-1$. By 4.11 , each $P_{i}, 1 \leq i<m$ is generated by a p-sequence of length $l+i$ and so the chain has length $h-l$. Thus $A_{n}$ is catenary. Goodearl and Lenagan $[5,3.13]$ have proved the catenarity of $A_{n}$ under the more general hypotheses that no $q_{i}$ is a root of unity.

(iv) We have seen that, under the hypotheses of 4.12, every prime factor $A_{n} / P$ of $A_{n}$ is a Noetherian domain with a localization isomorphic to the $k$-algebra $P(\Lambda)$ for some $m \times m$ matrix $\Lambda$ and some $m \geq 1$. Hence the quotient division ring $D$ of $A_{n} / P$ is isomorphic to the quotient division ring of the coordinate ring $A(\Lambda)$ of quantum $m$-space. Cauchon [3, II.2.1] has shown that if the group $H$ is torsion-free and $D$ is the quotient division ring of a prime factor of $A_{n}$ then there is a field extension $K$ of $k$ such that $D$ is isomorphic either to $K$ or the quotient division ring of the coordinate ring of quantum $m$-space over $K$ for some $m$.

(v) The analogue of Corollary 3.5 for $A_{n}$ is false. As the elements appearing in a p-sequence semicommute with each other, the nonmaximal prime ideals have the Artin-Rees property by [12, Theorem 4.2.7(i)]. However the maximal ideals do not have the Artin-Rees property and are not localizable. To see this, let $P=M+\left(y_{1}-\mu\right) A_{n}$ be a maximal ideal as in 4.12, and let $\alpha$ be the automorphism of $A_{n}$ induced by $z_{1}$, that is $z_{1} a=\alpha(a) z_{1}$ for all $a \in A_{n}$. Then $\alpha\left(y_{1}-\mu\right)=q_{1} y_{1}-\mu \notin P$. It follows from [8, Proposition 1] that $P$ does not have the Artin-Rees property and is not localizable.

\section{REFERENCES}

1. J. Alev and $F$. Dumas, Sur le corps des fractions de certaines algèbres quantiques, $J$. Algebra 170 (1994), 229-265.

2. V. W. Bryant, Aspects of combinatorics (Cambridge University Press, 1993).

3. G. Cauchon, Quotient premiers de $O_{q}\left(M_{n}(k)\right)$, preprint, Université de Reims.

4. K. R. Goodearl, Prime ideals in skew polynomial rings and quantized Weyl algebras, $J$. Algebra 150 (1992), 324-377.

5. K. R. Goodearl and T. H. Lenagan, Catenarity in quantum algebras, preprint, Universities of California (Santa Barbara) and Edinburgh.

6. K. R. Goodearl and E. S. Letzter, Prime ideals in skew and q-skew polynomial rings, Mem. Amer. Math. Soc. 109 (1994), no. 521.

7. K. R. Goodearl and R. B. Warfield, Jr., An introduction to noncommutative Noetherian rings (Cambridge University Press, 1989).

8. D. A. Jordan, Normal elements and completions of non-commutative Noetherian rings, Bull. London Math. Soc. 19 (1987), 417-424. 267-281.

9. D. A. Jordan, A simple localization of the quantized Weyl algebra, J. Algebra 174 (1995),

10. G. Maltsiniotis, Groupes quantique et structures différentielles, C.R. Acad. Sci. Paris Sér. I Math. 311 (1990), 831-834.

11. J. C. McConnell and J. J. Pettit, Crossed products and multiplicative analogues of Weyl algebras, J. London Math. Soc. (2) 38 (1988), 47-55. 
12. J. C. McConnell and J. C. Robson, Noncommutative Noetherian rings (Wiley, 1987).

13. S-Q. Oh, Primitive ideals of the coordinate ring of quantum symplectic space, J. Algebra 174 (1995), 531-552.

14. L. Rigal, Spectre de l'algèbre de Weyl quantique, preprint, Université de Paris VI (1994).

School of Mathematics and Statistics

Pure Mathematics Section

UNIVERSITY OF SHEFFIELD

THE HICKS BUILDING

SHEFFIELD S3 7RH

UK

Present address of first author:

Mathematics Department

IMAM KHOMEINI INTERNATIONAL UNIVERSTTY

GHAZVIN

ISLAMIC REPUBLIC OF IRAN 